\title{
Correction to: Conclusions and Future Research Direction in LSOPM
}

\section{Correction to:}

Chapter 5 in: H. Qudrat-Ullah and P. N. Panthallor, Operational Sustainability in the Mining Industry, Asset Analytics, https://doi.org/10.1007/978-981-15-9027-6_5

The original version of the book was inadvertently published with incorrect figure captions in Chapter 5, which have now been corrected. The book and the chapter have been updated with the changes. 